\title{
Feasibility of using retinol-binding protein from capillary blood specimens to estimate serum retinol concentrations and the prevalence of vitamin A deficiency in low-resource settings
}

\author{
Jonathan L Gorstein ${ }^{1, *}$, Omar Dary ${ }^{2}$, Pongtorn ${ }^{3}$, Bettina Shell-Duncan ${ }^{1}$, Tim Quick ${ }^{4}$ \\ and Emorn Wasanwisut ${ }^{3}$ \\ 'University of Washington, School of Public Health, Department of Health Services, Box 357660, H-688 Health \\ Sciences Center, Seattle, WA 98195-7660, USA: ${ }^{2}$ A2Z Project, Washington, DC, USA: ${ }^{3}$ Institute of Nutrition, \\ Mahidol University, Nakhon Pathom, Thailand: ${ }^{4}$ United States Agency for International Development (USAID), \\ Washington, DC, USA
}

Submitted 15 December 2006: Accepted 21 June 2007: First published online 17 0ctober 2007

\begin{abstract}
Vitamin A deficiency (VAD) is a significant public health problem in many countries. While cost-effective interventions are available to control VAD, reliable information is needed to the track progress of control programmes. However, assessment of VAD is uncommon because current approaches are expensive and not feasible in low-resource settings. The present study explores the utility of retinol-binding protein (RBP), analysed by enzyme-linked immunosorbent assay from capillary blood, as an alternative measure of serum retinol concentrations in populations. The study collected matched panels of venous and capillary blood from pre-school children in Chiang Mai, Thailand. Of a total sample of 195 children, there were no differences between RBP from venous blood, RBP from capillary blood or retinol from capillary blood relative to retinol from venous blood. Receiver-operating characteristic curve analysis suggested a cut-off of RBP $<0.825 \mu \mathrm{moll}^{-1}$ had optimal screening proficiency relative to retinol $<0.70 \mu \mathrm{moll}^{-1}$. For the purpose of population assessment, all three parameters performed well in screening for VAD relative to retinol from venous blood. There were no differences in the estimates of VAD between children stratified by inflammation status. Lower RBP concentrations were found in children in the early convalescent stage of infection than in children with no infection or in the late convalescent stage. This study provided evidence of the biological comparability between retinol and RBP estimated from venous blood and capillary blood. This is a critical observation as it provides empirical evidence that RBP from capillary blood is a surrogate measure of serum retinol concentrations.
\end{abstract}

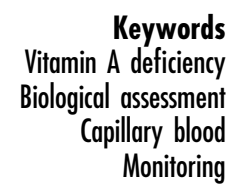

Vitamin A deficiency (VAD) is a significant public health problem in many countries and is the leading cause of preventable blindness throughout the world, particularly among children under 5 years of age. Recent estimates have shown that, globally, over 140 million people are affected by VAD, often leading to impaired immunity, xerophthalmia and even death ${ }^{1}$.

There are several cost-effective interventions which are being implemented in many countries to address VAD, including food fortification, dietary improvement and pharmacological supplementation. To identify the most appropriate intervention strategy, there is the need for reliable and accurate information on the magnitude and distribution of VAD in populations. Indeed, such information is also important to track changes in vitamin A
(VA) status following the introduction of interventions. Although assessment of VAD and VA status in populations is extremely important, it is not commonly done because current analytical methods are expensive, unreliable, and not appropriate in low-resource settings where VAD is most endemic ${ }^{2}$. As a result there is a scarcity of data on the prevalence of VAD, and, where interventions are in place, it has often not been possible to evaluate programme effectiveness.

The World Health Organization (WHO), the United Nations Children's Fund and the International Vitamin A Consultative Group (IVACG) have proposed a series of clinical and biochemical indicators to identify and monitor VAD in populations and to assess VA status ${ }^{3}$. In general, clinical indicators such as night blindness and 
other signs of xerophthalmia are rare among pre-school children and require very large sample sizes. These parameters are also prone to measurement variability and misclassification ${ }^{4,5}$. Biochemical indicators have also been recommended to identify populations with marginal VAD as well as compromised VA status, which itself has been found to be associated with an increased risk of mortality ${ }^{6,7}$.

The principal biochemical indicator of VA status recommended for international use has been serum or plasma retinol ${ }^{8}$. Retinol is usually assessed by highperformance liquid chromatography (HPLC), fluorometry or ultraviolet (UV) spectrophotometry. Although fluorometry and UV spectrophotometry are less expensive and require less sophisticated equipment than HPLC, the equipment is not as widely available as HPLC. While accepted as the internationally recommended indicator of VAD, the measurement of retinol by HPLC can be logistically complex, requiring sample volumes of $100 \mu \mathrm{l}$ and careful specimen collection, handling and storage. In addition, the maintenance of HPLC instrumentation has proved evasive in many settings. A proficiency test for laboratories measuring retinol and carotenoids reported that only seven out of the 16 participating developingcountry laboratories were able to measure serum retinol to an acceptable standard? .

Recently, retinol-binding protein (RBP) has been reported to correlate well with serum retinol in different population groups and has been found to be proficient in estimating the prevalence of low serum retinol concentrations among populations in several small field studies $^{10,11}$. A competitive enzyme immunoassay (EIA) has been developed which is significantly less expensive than HPLC and requires only standard equipment, such as a standard micro-well plate reader which may be available in many laboratories in developing countries currently doing EIA-based testing.

The collection of blood by venepuncture can be challenging in settings where it is not culturally appropriate and where some communities may be uncomfortable with the practice. Furthermore, there are field and safety constraints that limit the feasibility of venous blood collection. There has been a great deal of interest in assessing serum retinol concentrations using capillary blood, stored either in microcapillary tubes or as dried blood spots (DBS) on a filter paper matrix ${ }^{12,13}$ (also Hix J, Dried blood spots, Program for Appropriate Technology in Health (PATH) Laboratory Report, Notebook No. 0062, unpublished). While attractive for field use, the collection of capillary blood for assessment of retinol or RBP still requires cold chain conditions to ensure no degradation of specimen integrity. However, there are no published studies that specifically examine the biological equivalence between retinol and RBP from venous blood and from capillary blood. Thus the present study explores the utility of RBP, analysed by enzyme-linked immunosorbent assay from capillary blood, as an alternative measure of serum retinol concentrations in populations.

\section{Materials and methods}

The study was carried out in two remote districts of Chiang Mai, Thailand: Mae Cham District and Om Koi District. Data collection took place over a one-week period between the 3rd and 10th of September, 2004. In 2000-2001, there were reported cases of xerophthalmia among infants and young children in both districts and it was expected that $10-15 \%$ of the pre-school children would have marginal serum retinol levels. While these two districts are included in the Thailand national VAD control programme, the data enumeration for the study was planned to ensure that none of the children in these two districts had received a VA supplement within the previous five to six months. Supplements were provided in accordance with the national programme in late September. Matched panels of venous and capillary blood were taken from a cohort of 198 pre-school children. Serum from venous and capillary blood was assessed for retinol and RBP using HPLC and EIA, respectively. In addition, C-reactive protein (CRP) and $\alpha_{1}$-glycoprotein (AGP) were analysed from venous blood to control for inflammation ${ }^{14,15}$. Finally, haemoglobin levels of the children were measured in the field directly from capillary blood using a portable Hemo-Cue ${ }^{\mathrm{TM}}$ device.

The sample size for the study was estimated to be 200 children in order to characterise the distribution of biochemical indicators of VA and to analyse the correspondence between indicators using standard screening parameters. The sample size was calculated based on an expected screening level of $85 \%$ with absolute precision of $+5 \%$ and $95 \%$ confidence. ${ }^{*}$ Serum retinol from venous blood analysed by HPLC was taken as the reference and all other parameters were compared with this standard indicator.

The average age of the children in the study was 39 months, and ranged from 13 to 68 months of age. The sample was divided between the two districts of Mae Cham and Om Koi, and 100 children were recruited for each area. Local health workers developed a list of all children in the target age group and a sample was selected at random.

\footnotetext{
* The sample size calculation was based on the following formulas, where we assumed a screening index (sensitivity, $S n$; specificity, $S p$ ) of $85 \%$, a confidence level $\left(Z_{1-\alpha / 2}\right)$ of $95 \%$ (or 1.96) and a desired precision of $5 \%$, with $n$ being the total sample size:

$$
S n \pm Z_{1-\alpha / 2} \sqrt{\frac{S n(1-S n)}{n}} ; \quad S p \pm Z_{1-\alpha / 2} \sqrt{\frac{S p(1-S p)}{n}} .
$$
}


Ethical and human subjects' clearance for the study was obtained from the Scientific Committee of the Institute of Nutrition at Mahidol University (INMU, Nakhon Pathom, Thailand) and from the Program for Appropriate Technology in Health (PATH, Seattle, WA, USA). Consent for the study was also obtained from provincial and district health authorities following a series of meetings with representatives of INMU and the Nutrition Division of the Ministry of Health. Local government health officials from the District Health Office and the Primary Care Unit approached the mothers of the children, explained the objectives of the study, and requested consent for a physical check-up and the participation of the children in the study. Before the start of the physical examination, written consent was requested from all parents/guardians who agreed for their child to participate. The parents were asked to have their children gather at a central data collection location where data enumeration, including the collection of biological specimens, was handled systematically and in a controlled manner.

A questionnaire was administered to collect basic demographic data of all participating children, including age and gender. Clinical examinations focusing on acute diarrhoea and respiratory illness were undertaken by trained medical personnel to ascertain current status of infectious morbidity. After the administration of the questionnaire and the physical examination, each child and the child's mother were brought into a private blood collection room where blood samples were taken.

Nurses first drew venous blood with a Vacutainer Blood Collection Set or a Sarstedt Multi-Fly Set (Becton Dickinson and Co.). The volume of venous blood collected was approximately $2 \mathrm{ml}$ from each child. Each tube was labelled with an individual identification code. The nurse then collected capillary blood by finger puncture using disposable lancets (BD Genie). Free-flowing capillary blood was collected after wiping away the first blood drop, with the second blood drop used to fill a cuvette for analysis of haemoglobin in the portable HemoCue $\mathrm{TM}^{\mathrm{TM}}$ device. On average, five or six capillary tubes of blood were collected from each child. In some cases a second finger puncture was required in order to retrieve sufficient volume of capillary blood. The capillary tubes (VITREX BRIS MicroHaematocrit Tubes, ref. 160231, ISO 12772, Plain, Nonheparinized; MODULOHM A/S) were plugged with clay, and tape was placed on these with the individual codes. The blood was allowed to clot for $40 \mathrm{~min}$ at room temperature and then centrifuged. Venous blood was centrifuged with a Compact Lab Centrifuge (model Z150A; Labnet) at $3500 \mathrm{rpm}$ for $15 \mathrm{~min}$, while capillary blood was centrifuged with a Hettich 2010 or DAMON/IEC (model MB) for $7 \mathrm{~min}$. Serum from venous blood was separated into two amber microtubes, the first containing at least $0.5 \mathrm{ml}$ and the second containing $0.2 \mathrm{ml}$.
Serum from capillary blood was collected by cutting the capillary tubes with a file, and two aliquots of at least $50 \mu \mathrm{l}$ of serum were transferred to individual PCR (polymerase chain reaction) tubes. At the end of each day of data collection the tubes were labelled with code numbers, placed in storage boxes, and then placed in ice pack boxes and transported to Mae Cham Hospital or Om Koi Hospital. These samples were maintained in the hospital's freezer at $-20^{\circ} \mathrm{C}$. When data collection was completed, all specimens were shipped frozen to the laboratory at INMU for analysis. RBP was analysed from serum of both specimen types using the PATH RBP-EIA test (Scimedx). Retinol was analysed from both capillary and venous serum by conventional HPLC, while CRP and AGP were measured from serum of the venous specimens by radial immunodiffusion (Kent Laboratories). All analytes were analysed within three weeks of data collection in the field.

Capillary and venous serum samples were analysed for both retinol and RBP. First, both specimens and calibrator sera were diluted 1:25 in assay buffer in a low proteinbinding micro-well plate. Next, the specimens and calibrator sera were mixed by reverse pipetting (VortexGenie 2; Scientific Industries, Inc.) and transferred to test wells in plastic low protein-binding micro-well plates (Evergreen Medical Products). One hundred microlitres of monoclonal anti-RBP antibody conjugated to horseradish peroxidase enzyme were then immediately added to each sample test well. The plates were incubated for $15 \mathrm{~min}$ at room temperature $\left(18-25^{\circ} \mathrm{C}\right)$; after $10 \mathrm{~min}$ the sample wells were mixed by gently tapping the plate frame.

At the end of the 15-min incubation period, test plates were inverted and emptied by flicking the contents into a sink. The test wells were then washed five times using wash buffer. Immediately following the final wash, $200 \mu \mathrm{l}$ of 3,3',5,5'-tetramethylbenzidine (Moss Ultra-sensitive) containing hydrogen peroxide substrate was added to each well. The plates were incubated at room temperature $\left(18-25^{\circ} \mathrm{C}\right)$ for another $10 \mathrm{~min}$, mixed briefly at $5 \mathrm{~min}$ and again near the end of the incubation by gently tapping the plate frame. Following this incubation, $100 \mu \mathrm{l}$ of stop solution was added to all of the test wells. The plates were immediately read using the EIA plate reader (Sunrise; Tecan Austria GmbH) fitted with a $450 \mathrm{~nm}$ filter, and the resulting optical densities were recorded using Magellan software version 3.12 (Tecan). The results from the analysis were compared with values obtained from the calibrator sera by linear regression software or by plotting the RBP concentration against the optical density obtained from the calibrator sera. The tests were run in duplicate and the intra-assay coefficient of variation was $<5 \%$ in all samples.

The samples were thawed, an aliquot of $50 \mu \mathrm{l}$ was extracted in hexane, and $20 \mu l$ were injected by an autosampler (Waters 717plus) onto an analytical column and 
analysed by reverse-phase HPLC (Waters Corp.) ${ }^{16}$. Retinol was detected using a programmable UV-visible detector (Waters 486) that was set at $325 \mathrm{~nm}$. Retinol was separated using a Resolve ${ }^{\mathrm{TM}}$ C18 analytical column (150.0 $\mathrm{mm} \times$ $3.9 \mathrm{~mm}$ diameter; Waters Corp.) containing $5 \mu \mathrm{m}$ particles protected with a C18 guard column $2.0 \mathrm{~mm} \times 20.0 \mathrm{~mm}$ (Upchurch Scientific, Inc.). Isocratic analyses were performed at a flow rate of $0.7 \mathrm{ml} \mathrm{min}^{-1}$ with a mobile phase of absolute methanol that was delivered by a Waters 515 dual-head pump. The data for each determination were transmitted, stored and processed by a personal computer using Millennium Workstation software (version 3.05.01; Waters Corp.).

\section{Statistical metbods}

Four parameters of VA status were calculated. Data were examined for normal distribution characteristics using the Kolmogorov-Smirnov test. To classify VAD, the cut-off point of $0.70 \mu \mathrm{moll}^{-1}$ as recommended by WHO and IVACG was used for all parameters. In addition, a receiver-operating characteristic (ROC) curve was plotted to determine the optimal correspondence between different cut-off criteria for RBP from venous blood in classifying VAD as compared with retinol $<0.70 \mu \mathrm{moll}^{-1}$. The ROC curve provides a graphic representation of the balance between sensitivity and specificity of a diagnostic tool relative to a reference standard. That is, any increase in sensitivity will be accompanied by a decrease in specificity. The closer the curve follows the left vertical axis and then the top horizontal axis of the ROC space, the more accurate the test. In turn, the area under the curve is a measure of the overall accuracy of the diagnostic test parameter.

To assess inflammation, cut-off points were used for $\mathrm{CRP}\left(>5.0 \mathrm{mgl}^{-1}\right)$ and for AGP $\left(>1.0 \mathrm{gl}^{-1}\right)$ as recommended by Thurnham et al. ${ }^{14}$. Based on these criteria the status of inflammation was classified to reflect the stage of infection as: (1) normal - no elevated acute-phase proteins; (2) incubation - elevated CRP, but normal AGP; (3) early convalescence - both acute-phase proteins elevated; and (4) late convalescence - elevated AGP but normal CRP. For the classification of anaemia, the cut-off of $\mathrm{Hb}<11 \mathrm{~g} \mathrm{dl}^{-1}$ was designated as recommended by WHO for pre-school children ${ }^{17}$.

Differences in prevalence of VAD based on the different indicators were measured by Pearson's $\chi^{2}$ test. Differences in the distribution of indicators between groups of children with different inflammation status were analysed using Student's paired $t$-test. Linear regression models were plotted to establish the correlation between the different parameters of VA status. Statistical analysis was carried out using SPSS 12.0 (SPSS Inc.). The sensitivity, specificity, positive predictive value and negative predictive value were calculated relative to retinol from venous blood using the cut-off point of
$<0.70 \mu \mathrm{mol}^{-1}$ taken to reflect the reference standard for the classification of VAD.

\section{Results}

Of the total sample of 200 children, 198 were given parental consent to participate in the study. Of the 198, venous blood could not be collected from two children, while the capillary blood from another child was not available as the capillary tube broke when it was being centrifuged in the field. There were, therefore, totals of 196 venous samples and 195 capillary blood specimens collected, and a matched panel was available for a cohort of 195 children. There was sufficient volume of both capillary blood and venous blood from all 195 children to undertake all laboratory analyses as per the study protocol.

Table 1 provides general demographic data of the study population. There was equal representation from both of the study districts, and the gender balance was also equivalent. There was an effort to ensure that preschool children between 12 and 59 months of age were included in the cohort, and this was realised with some 93\% of all children falling in this range, including some $21.7 \%$ under 2 years of age. It was possible to collect an adequate volume of capillary blood from these younger children.

An ROC curve was plotted to assess the most appropriate cut-off point for RBP relative to retinol with $<0.70 \mu \mathrm{mol}$ retinol $\mathrm{l}^{-1}$ taken as the reference criterion for VAD. While it has been presumed that the same cut-off point used to classify VAD based on retinol be used for RBP based on the assumption that there is a 1:1 molar relationship between these compounds, other studies have noted that a higher cut-off point achieves greater proficiency ${ }^{10,11}$. Indeed, in this study the ROC curve analysis suggested that a cut-off point of $<0.825 \mu \mathrm{mol}$ RBP $1^{-1}$ had the most optimal screening proficiency (area under curve $=0.011$ ) relative to retinol $<0.70 \mu \mathrm{moll}^{-1}$. Table 2 presents data on the biological parameters,

Table 1 Basic characteristics of the study population

\begin{tabular}{lrr}
\hline Parameter & $n$ & $\%$ \\
\hline District & & \\
Om Koi & 100 & 50.5 \\
Mae Cham & 98 & 49.5 \\
Gender & 102 & \\
$\quad$ Boys & 96 & 48.5 \\
Girls & & \\
Age (months) & 43 & 21.7 \\
12-24 & 42 & 21.2 \\
$24-36$ & 54 & 27.3 \\
$36-48$ & 45 & 22.7 \\
$48-60$ & 14 & 7.1 \\
$>60$ & & \\
\hline
\end{tabular}


Table 2 Biological parameters of the study population

\begin{tabular}{|c|c|c|c|c|c|}
\hline Parameter & Mean & SD & Cut-off & $\%$ of values below cut-off & $95 \% \mathrm{Cl}$ \\
\hline Retinol-venous ( $\left.\mu \mathrm{moll}^{-1}\right)$ & 1.063 & 0.26 & $<0.70 \mu \mathrm{moll}^{-1}$ & 7.2 & $3.6-10.8$ \\
\hline Retinol-capillary $\left(\mu \mathrm{moll}^{-1}\right)$ & 1.076 & 0.29 & $<0.70 \mu \mathrm{moll}^{-1}$ & 7.7 & $3.9-11.4$ \\
\hline RBP-venous $\left(\mu \mathrm{moll}^{-1}\right)$ & 1.121 & 0.22 & $<0.82 \mu \mathrm{moll}^{-1}$ & 7.7 & $3.9-11.4$ \\
\hline RBP-capillary ( $\left.\mu \mathrm{mol}^{-1}\right)$ & 1.118 & 0.23 & $<0.82 \mu \mathrm{moll}^{-1}$ & 9.2 & $5.1-13.2$ \\
\hline $\mathrm{Hb}\left(\mathrm{g} \mathrm{dl}^{-1}\right)$ & 11.65 & 1.43 & $<11 \mathrm{~g} \mathrm{dl}^{-1}$ & 28.4 & $22.1-34.7$ \\
\hline $\operatorname{AGP}\left(\mathrm{gI}^{-1}\right)$ & 0.916 & 0.33 & $>1.0 \mathrm{gl}^{-1}$ & 24.0 & $18.0-30.0$ \\
\hline $\operatorname{CRP}\left(\mathrm{mgl}^{-1}\right)$ & 1.39 & 4.42 & $>5 \mathrm{mgl}^{-1}$ & 9.7 & $5.6-13.8$ \\
\hline \multicolumn{6}{|l|}{ Inflammation status* } \\
\hline Healthy & & & & 66.3 & $59.7-72.9$ \\
\hline Incubation & & & & 1.0 & $0.1-3.6$ \\
\hline Early convalescence & & & & 8.7 & $5.1-13.5$ \\
\hline Late convalescence & & & & 24.0 & $18.2-30.6$ \\
\hline
\end{tabular}

$\mathrm{SD}$ - standard deviation; $\mathrm{Cl}$ - confidence interval; RBP - retinol-binding protein; $\mathrm{Hb}$ - haemoglobin; AGP - $\alpha_{1}$-glycoprotein; CRP - C-reactive protein. ${ }^{*}$ Healthy - no raised acute-phase proteins; Incubation - CRP $>5 \mathrm{mgl}^{-1}, A G P \leq 1.0 \mathrm{gl}^{-1}$; Early convalescence $-\mathrm{CRP}>5 \mathrm{mgl}^{-1}, A G P>1.0 \mathrm{gl}^{-1}$; Late convalescence $-\mathrm{CRP} \leq 5 \mathrm{mg}^{-1}, \mathrm{AGP}>1.0 \mathrm{gl}^{-1}$.

Table 3 Proficiency in estimating low serum retinol by different parameters, controlling for inflammation status* (all comparisons are relative to retinol-venous $<0.70 \mu \mathrm{moll}^{-1}$ )

\begin{tabular}{|c|c|c|c|c|c|c|c|}
\hline \multirow[b]{2}{*}{ Parameter } & \multirow[b]{2}{*}{ Cut-off } & \multicolumn{2}{|c|}{ All children $(n=196)$} & \multicolumn{2}{|c|}{ Early convalescence $(n=17)$} & \multicolumn{2}{|c|}{ Late convalescence $(n=47)$} \\
\hline & & Sensitivity (\%) & Specificity (\%) & Sensitivity (\%) & Specificity (\%) & Sensitivity (\%) & Specificity (\%) \\
\hline Retinol-capillary & $<0.70 \mu \mathrm{mol}^{-1}$ & 71.4 & 97.2 & 100.0 & 76.9 & 66.7 & 100.0 \\
\hline RBP-venous & $<0.825 \mu \mathrm{mol}^{-1}$ & 92.9 & 98.9 & 75.0 & 92.3 & 100.0 & 100.0 \\
\hline RBP_capillary & $<0.825{\mu \mathrm{mol}^{-1}}^{-1}$ & 85.7 & 96.7 & 75.0 & 92.3 & 100.0 & 97.7 \\
\hline
\end{tabular}

RBP - retinol-binding protein.

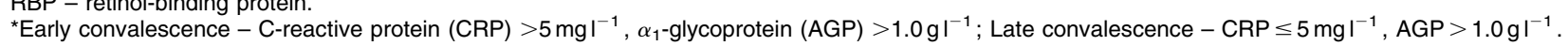

anaemia and inflammation status in the study population. The prevalence of anaemia in this study population was $28.4 \%$ (95\% confidence interval (CI) 22.1-34.7). The mean levels were slightly lower for retinol than for RBP with virtually no difference between the capillary and venous blood samples for each parameter. All four VA parameters followed normal distributions and had similar variance estimates. The prevalence of VAD based on serum retinol from venous blood using the cut-off point of $<0.70 \mu \mathrm{moll}^{-1}$ was $7.2 \%$ (95\% CI 3.6-10.8), while the prevalence was slightly higher when using retinol from capillary blood $(7.7 \%$, 95\% CI 3.9-11.4). Using a cut-off point for RBP of $0.825 \mu \mathrm{moll}^{-1}$ as suggested by the ROC curve analysis, the prevalence of VAD was $7.7 \%$ from venous blood (95\% CI 3.9-11.4) and 9.2\% from capillary blood (95\% CI 5.1-13.2). The differences in VAD prevalence between the four parameters did not reach statistical significance.

Table 3 outlines the screening proficiency of the three VA parameters against retinol analysed from venous blood. Not surprisingly, given the low prevalence of VAD observed in the study population, the specificity of all parameters was very high, all above 96.5\%. For retinol from capillary blood, the sensitivity was $71.4 \%$. Using a cut-off point of $0.825 \mu^{m o l}{ }^{-1}$ for RBP, the sensitivity of the RBP from venous blood was $92.9 \%$ while the sensitivity of capillary blood was $85.7 \%$. For purposes of population assessment, all three test parameters seemed to perform as well as one another in screening for VAD relative to retinol from venous blood.

There was a significant association between CRP and AGP. There was a 20-fold increased probability of a child having elevated CRP if $\mathrm{s} /$ he also had elevated AGP. In fact, of the 19 children with elevated CRP, 17 also had high AGP levels indicating that most children had advanced from the incubation stage of infection to early convalescence. Table 4 presents data showing the association between inflammation and VAD.

There were no differences in the estimates of VAD within each cohort of children stratified by inflammation status. That is, all four parameters responded in the same manner to different stages of infection. There was a dramatically lower RBP and retinol among those children in the early convalescent stage of infection than in children with no infection or in the late convalescent stage. However, the prevalence of low retinol from venous blood among children in the early convalescent stage of illness (41.2\%; 95\% CI 18.4-67.1) was significantly higher than among children with no inflammation (4.6\%; 95\% CI 1.7-9.8) and among children in later convalescence (4.3\%, 95\% CI 0.5-14.5). 
Table 4 Prevalence (\%) of low serum retinol and low RBP, stratified by inflammation status* (exact 95\% Cl based on binomial distribution)

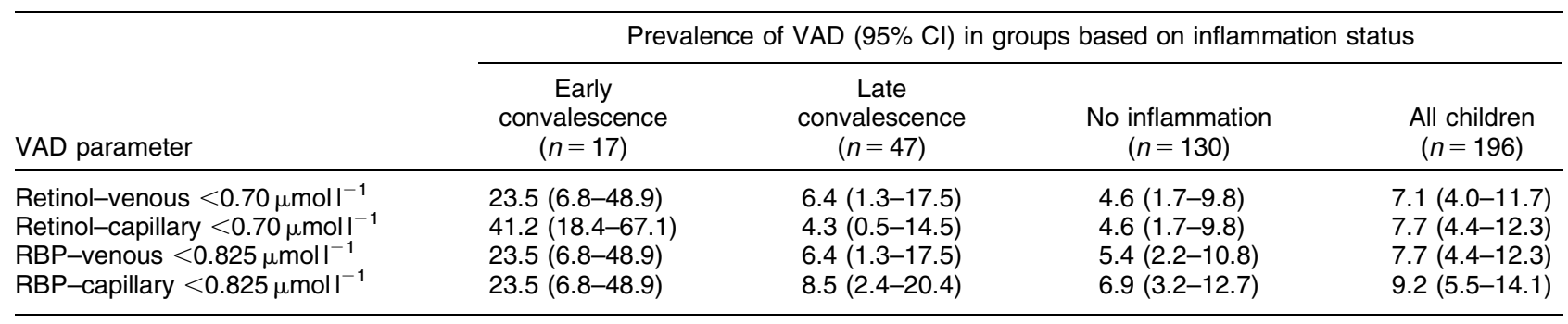

RBP - retinol-binding protein; $\mathrm{Cl}$ - confidence interval; VAD - vitamin A deficiency.

${ }^{*}$ Early convalescence - C-reactive protein (CRP) $>5 \mathrm{mgl}^{-1}, \alpha_{1}$-glycoprotein (AGP) $>1.0 \mathrm{gl}^{-1}$; Late convalescence $-\mathrm{CRP} \leq 5 \mathrm{mgI}^{-1}, \mathrm{AGP}>1.0 \mathrm{gl}^{-1}$; No inflammation - no elevated acute-phase proteins.

Table 5 Comparison between methods of VAD assessment, stratified by inflammation status ${ }^{*}$ - retinol and RBP

\begin{tabular}{|c|c|c|c|c|c|}
\hline \multirow[b]{3}{*}{ Parameter } & \multicolumn{5}{|c|}{ Retinol $\left(\mu \mathrm{moll}^{-1}\right)$} \\
\hline & \multicolumn{2}{|c|}{ Venous } & \multicolumn{2}{|c|}{ Capillary } & \multirow[b]{2}{*}{$P$-value } \\
\hline & Mean & SD & Mean & SD & \\
\hline All children $(n=196)$ & 1.06 & 0.26 & 1.07 & 0.29 & 0.356 \\
\hline Early convalescence $(n=17)$ & 0.86 & 0.25 & 0.80 & 0.28 & 0.148 \\
\hline Late convalescence $(n=47)$ & 1.11 & 0.31 & 1.12 & 0.33 & 0.672 \\
\hline \multirow[t]{3}{*}{ No inflammation $(n=130)$} & 1.08 & 0.23 & 1.10 & 0.25 & 0.232 \\
\hline & \multicolumn{5}{|c|}{$\operatorname{RBP}\left(\mu \mathrm{moll}^{-1}\right)$} \\
\hline & \multicolumn{2}{|c|}{ Venous } & \multicolumn{2}{|c|}{ Capillary } & \\
\hline Parameter & Mean & SD & Mean & SD & $P$-value \\
\hline All children $(n=196)$ & 1.12 & 0.22 & 1.12 & 0.23 & 0.873 \\
\hline Early convalescence $(n=17)$ & 0.95 & 0.21 & 0.94 & 0.21 & 0.677 \\
\hline Late convalescence $(n=47)$ & 1.13 & 0.22 & 1.13 & 0.24 & 0.823 \\
\hline No inflammation $(n=130)$ & 1.14 & 0.21 & 1.14 & 0.22 & 0.806 \\
\hline
\end{tabular}

VAD - vitamin A deficiency; RBP - retinol-binding protein; SD - standard deviation.

${ }^{*}$ Early convalescence $-\mathrm{C}$-reactive protein (CRP) $>5 \mathrm{mgI}^{-1}, \alpha_{1}$-glycoprotein (AGP) $>1.0 \mathrm{gl}^{-1}$; Late convalescence - CRP $\leq 5 \mathrm{mgl}^{-1}$, AGP $>1.0 \mathrm{gl}^{-1}$; No inflammation - no elevated acute-phase proteins.

There were no differences between the mean retinol values or mean RBP values from venous blood and capillary blood for all groups of children when accounting for infection (Table 5). The mean values were lower for those children in the early convalescence stage, but both venous and capillary blood followed identical patterns.

There were also no differences between the mean RBP values from capillary and venous blood among all children, as well as when stratified by inflammation status. In fact, the correspondence between the RBP from venous and capillary blood seemed to be even stronger than seen with retinol. As expected, the overall mean levels of RBP were slightly higher than those for retinol.

\section{Discussion and conclusions}

This study provided evidence of the biological comparability between serum retinol levels estimated from venous blood and capillary blood. This is a critical observation as it provides empirical evidence that this important indicator of VA status may be measured from capillary blood, which has important implications for non-clinical population-based research. Finger-prick sampling collection is relatively painless and non-invasive, and capillary blood sampling eliminates the need for a trained phlebotomist to collect samples. It should be noted that for this study serum was the sample type that was used, and it was not possible to examine whether similar results would have been realised had plasma been separated from the venous blood and capillary blood before being subjected to HPLC analysis for retinol determination.

It is important to mention that while retinol is the internationally recommended indicator to assess the prevalence of VAD at the population level, it is not a direct measure of hepatic stores of $\mathrm{VA}^{18,19}$. There should be careful interpretation of these estimates as a reflection of the total VA pools in the body ${ }^{20}$. At the same time, there are no viable alternative indicators of VA stores, so the use of RBP as a surrogate indicator to estimate low serum retinol concentrations has important practical relevance for programmes.

This study adds to a growing body of research that has documented a significant linear correlation between retinol and RBP in serum derived from venous blood samples $^{10,11}$. Further, the results from this study demonstrated a close correspondence between retinol in venous blood samples and RBP in capillary blood. Moreover, serum RBP, often seen as a surrogate measure for serum retinol, had a significant screening proficiency in classifying low serum retinol concentrations among populations. These studies, which have examined the correspondence between retinol and RBP, have suggested that a slightly higher molar cutoff point for RBP should be used when estimating VAD than is employed for retinol. ROC curve analysis from this study indicated that a cut-off point of RBP $<0.825 \mu \mathrm{moll}^{-1}$ should be used to estimate VAD prevalence in comparison with the cut-off point of retinol $<0.70 \mu \mathrm{moll}^{-1}$. Because many programme managers responsible for the implementation of VAD control are most concerned with changes in the prevalence of $\mathrm{VAD}$ in response to interventions, 
this study provides some clear avenues for simplifying specimen collection and laboratory analysis to support programme management.

The study also considered the association between retinol and RBP from venous and capillary specimens, controlling for inflammation. Infectious diseases cause an increase in acute-phase proteins such as CRP and AGP. The relative concentration of these two acute-phase proteins indicates the duration of inflammation, where CRP is raised in the initial hours following infection and reaches its highest levels within two days and normalises rapidly. In contrast, AGP takes longer to rise and does not reach its highest levels until several days following the infection and remains elevated during convalescence ${ }^{21}$. As has been seen in other studies, there was a strong association between retinol and RBP with inflammation status, particularly in the stage of early convalescence. The fact that the same pattern was not as strong in late convalescence suggests that CRP in this study is a more important parameter in this population in confounding the assessment of VA status. It is important to note that not all children with low retinol or low RBP values in the presence of inflammation may be VA-deficient, and the interpretation of prevalence data must be done with caution $^{15}$.

It has been suggested that one way to handle the depressed parameters of VA status in the presence of inflammation is to exclude those children with inflammation when generating estimates of VAD. The basis for this recommendation is that by only including healthy children in the analysis, low retinol or RBP will reflect overt VAD rather than depressed VA that may be confounded by inflammation. However, this could potentially bias a sample ${ }^{22}$ and not provide a true representation of VAD in a population as children with VAD are more likely to be suffering from infection. Others have recommended the use of correction factors to adjust VAD prevalence to account for inflammation in a population ${ }^{23}$, but these factors have not been refined. It has also been suggested that the ratio of RBP to transthyretin may be used to assess VA status during inflammation among children ${ }^{24}$. Future research is required to better answer these critical questions of data interpretation.

For this study, which aimed to establish the underlying biological relationships between retinol and RBP from different specimen types, capillary blood was collected and stored using microcapillary tubes. It is important to note that capillary tubes still require controlled conditions for sample processing and transportation that may be difficult to attain in the field. One possible alternative may be to use filter paper matrices for storing capillary blood as DBS. DBS methods are available for a growing number of analytes including CRP, thyroid hormones, retinol and serum transferrin receptor ${ }^{25}$. Several community-based applications have shown this collection method to be a reliable means of simplifying sample storage and transport. Further research will, however, be necessary to examine the most appropriate and fieldfriendly methods of collecting capillary blood.

The finding of a strong correlation between retinol and RBP in both venous and capillary samples suggests that investigators have additional options when assessing low serum retinol concentrations in community-based studies. The analysis of RBP from capillary blood has many advantages, including ease in sample collection, accuracy in detection of VAD, affordability, and feasible analysis with standard laboratory equipment. The information derived from this study about the feasibility of using capillary blood for assessing VA status is exciting, yet it is important to emphasise that great measures were taken to ensure that pristine specimens were collected and vigilant efforts were followed to maintain rigor in specimen handling and storage.

\section{Acknowledgements}

Sources of funding: This study was funded in part by the Agency for International Development under the HealthTech Project of the Program for Appropriate Technology in Health (PATH).

Conflict of interest declaration: Although the study received funding from $\mathrm{PATH}$ and the study included the analysis of RBP using the RBP-EIA developed by PATH, all research protocols and study implementation were executed independently of PATH to ensure that there were no compromises to the integrity of the work or the objectivity of the results.

Authorship responsibilities: J.L.G. served as principal investigator and assisted in the design, implementation and analysis of the study, and was the lead author on the paper. O.D. provided technical assistance on the study design, with particular emphasis on the relevance of parameters of inflammation, and provided major input to the initial draft of the paper. P. was responsible for all laboratory analyses of capillary and venous specimens, as well as data analysis. B.S.-D. was responsible for study design and training of fieldworkers in enumeration and collection of biological specimens, and supported data collection and transport of specimens to the laboratory. T.Q. provided overall assistance in the study design, data analysis and preparation of the article. E.W. provided assistance in study design, and was responsible for the supervision of all field activities and laboratory analyses.

\section{References}

1 Darnton-Hill I, de Pee S, Bloom MW. New issues in developing effective approaches for the prevention and control of vitamin A deficiency. Food and Nutrition Bulletin 1998; 19: 137-46. 
2 de Pee S, Dary O. Biochemical indicators of vitamin A deficiency: serum retinol and serum retinol binding protein. Journal of Nutrition 2002; 132: 2895S-901S.

3 World Health Organization (WHO). Indicators for Assessing Vitamin A Deficiency and their Application in Monitoring and Evaluating Intervention Programs. WHO/NUT/96.10. Geneva: WHO, 1996.

4 Arroyave G, Chichester CO, Flores H, Glover J, Mejia LA, Olson JA, et al. Biochemical Methodology for the Assessment of Vitamin A Status. Washington, DC: International Vitamin A Consultative Group, 1982.

5 Arroyave G, Baltazar J, Kusin J, Lepkowski JM, Milton RC, Srikantia SG. Methodologies for Monitoring and Evaluating Vitamin A Deficiency Intervention Programs. Washington, DC: International Vitamin A Consultative Group, 1989.

6 Sommer A, Hussaini G, Tarwotjo I, Susanto D. Increased mortality in children with mild vitamin A deficiency. Lancet 1983; 2: 585-8.

7 Milton RC, Reddy V, Naidu AN. Mild vitamin A deficiency and childhood morbidity - an Indian experience. American Journal of Clinical Nutrition 1987; 46: 827-9.

8 Olson JA. Vitamin A, retinoids and carotenoids. In: Shils ME, Olson JA, Shike M, eds. Modern Nutrition in Health and Disease. Philadelphia, PA: Lea \& Febiger, 1994; 287-307.

9 Hulshof PJM, Brouwer JT, Burema J, West CE. Bias and random error in retinol measurements of laboratories in countries with populations with mild to severe vitamin A deficiency. Clinical Chemistry 2002; 48: 2061-3.

10 Gamble MV, Ramakrishnan R, Palafox NA, Briand K, Berglund L, Blaner WS. Retinol binding protein as a surrogate measure for serum retinol: studies, in vitamin Adeficient children from the Republic of the Marshall Islands. American Journal of Clinical Nutrition 2001; 73: 594-601.

11 Almekinder J, Manda W, Soko D, Lan Y, Hoover DR, Semba RD. Evaluation of plasma retinol-binding protein as a surrogate measure for plasma retinol concentrations. Scandinavian Journal of Clinical and Laboratory Investigation 2000; 60: 199-204.

12 Mirchamsy H, Nazari F, Stellman C, Esterabady H. The use of dried whole blood absorbed on filter paper for the evaluation of diphtheria and tetanus antitoxins in mass survey. Bulletin of the World Health Organization 1968; 38 : $665-71$.

13 Craft NE, Bulux J, Valdez C, Li Y, Solomons NW. Retinol concentrations in capillary dried blood spots from healthy volunteers: method validation. American Journal of Clinical Nutrition 2000; 72: 450-4

14 Thurnham DI, McCabe GP, Northrop-Clewes CA, Nestel P. Effects of subclinical infection on plasma retinol concentrations and assessment of prevalence of vitamin A deficiency: meta-analysis. Lancet 2003; 362: 2052-8.
15 Stephensen CB, Gildengorin G. Serum retinol, the acute phase response, and the apparent misclassification of vitamin A status in the third National Health and Nutrition Examination Survey. American Journal of Clinical Nutrition 2000; 72: 1170-8.

16 Sowell AL, Huff DL, Yeager PR, Caudill SP, Gunter EW. Retinol, $\alpha$-tocopherol, lutein/zeaxanthin, $\beta$-cryptoxanthin, lycopene, $\alpha$-carotene, trans- $\beta$-carotene, and four retinyl esters in serum determined simultaneously by reversedphase HPLC with multiwavelength detection. Clinical Chemistry 1994; 40: 411-16.

17 World Health Organization (WHO). Iron Deficiency Anaemia: Assessment, Prevention and Control. WHO/NHD/01.3. Geneva: WHO, 2001.

18 Haskell MJ, Mazumder RN, Peerson JM, Jones AD, Wahed MA, Mahalanabis D, et al. Use of the deuteratedretinol-dilution technique to assess total-body vitamin A stores of adult volunteers consuming different amounts of vitamin A. American Journal of Clinical Nutrition 1999; 70: 874-80.

19 Haskell MJ, Handelman GJ, Peerson JM, Jones AD, Rabbi A, Awal MA, et al. Assessment of vitamin A status by the deuterated retinol dilution technique and comparison with hepatic retinol concentration in Bangladeshi surgical patients. American Journal of Clinical Nutrition 1997; 66 : $67-74$.

20 Furr HC, Amedee-Manesme O, Clifford AJ, HR, Jones AD, Anderson DP, et al. Vitamin A concentrations in liver determined by isotope dilution assay with tetradeuterated vitamin A and by biopsy in generally healthy adult humans. American Journal of Clinical Nutrition 1989; 49: 713-16.

21 Fleck A, Myers MA. Diagnostic and prognostic significance of the acute-phase proteins. In: Gordon AH, Koj A, eds. The Acute-Phase Response to Injury and Infection. Amsterdam: Elsevier Science Publishers BV, 1985; 249-71.

22 Maqsood M, Dancheck B, Gamble M, Palafox NA, Ricks $\mathrm{MO}$, Briand $\mathrm{K}$, et al. Vitamin A deficiency and inflammatory markers among preschool children in the Republic of the Marshall Islands. Nutrition Journal 2004; 3: 21.

23 Wieringa FT, Dijkhuizen MA, West CE, Northrop-Clewes CA, Muhilal. Estimation of the effect of the acute phase response on indicators of micronutrient status in Indonesian infants. Journal of Nutrition 2002; 132: 3061-6.

24 Filteau SM, Willumsen JF, Sullivan K, Simmank K, Gamble $M$. Use of the retinol-binding protein:transthyretin ratio for assessment of vitamin A status during the acute-phase response. British Journal of Nutrition 2000; 83: 513-20.

25 McDade TW, Shell-Duncan B. Whole blood collected on filter paper provides a minimally invasive method for assessing human transferrin receptor level. Journal of Nutrition 2002; 132: 3760-3. 